University of Wollongong

Research Online

Faculty of Social Sciences - Papers (Archive) Faculty of Arts, Social Sciences \& Humanities

2020

Exploring stakeholders' perceptions of the acceptability, usability, and dissemination of the australian 24-hour movement guidelines for the early years

Rebecca M. Stanley

University of Wollongong, rstanley@uow.edu.au

Rachel A. Jones

University of Wollongong, rachelj@uow.edu.au

Christian F. Swann

cswann@uow.edu.au

Hayley Christian

Julie Sherring

University of Wollongong

See next page for additional authors

Follow this and additional works at: https://ro.uow.edu.au/sspapers

Part of the Education Commons, and the Social and Behavioral Sciences Commons

Research Online is the open access institutional repository for the University of Wollongong. For further information contact the UOW Library: research-pubs@uow.edu.au 


\title{
Exploring stakeholders' perceptions of the acceptability, usability, and dissemination of the australian 24-hour movement guidelines for the early years
}

\author{
Abstract \\ Background: Australian 24-Hour Movement Guidelines for the Early Years were recently developed. To \\ maximize the uptake of the guidelines, perceptions of key stakeholders were sought. Methods: Thirty-five \\ stakeholders (11\% Aboriginal or Torres Strait Islander descent) participated in focus groups or key \\ informant interviews. Stakeholders included parents of children aged 0-5 years, early childhood \\ educators, and health and policy professionals, recruited using convenience and snowballing techniques. \\ Focus groups and interviews were audio-recorded and transcribed verbatim. Data were analyzed \\ inductively using thematic analysis. Results: There was general acceptance of the Movement Guidelines. \\ The stakeholders suggested that the Guidelines were highly aspirational and needed to be carefully \\ messaged, so parents did not feel guilty if their child was not meeting them. Stakeholders identified that \\ the messaging needed to be culturally appropriate and visually appealing. Dissemination strategies \\ differed depending on the stakeholder. Conclusion: Seeking stakeholder perceptions is an important \\ process in the development of national Movement Guidelines. This study successfully examined \\ stakeholders' perceptions regarding the acceptability, usability, and dissemination of the Australian \\ 24-Hour Movement Guidelines. Effective and innovative strategies for maximizing compliance and uptake \\ of the Guidelines should be prioritized. \\ Disciplines \\ Education | Social and Behavioral Sciences

\section{Publication Details} \\ Stanley, R., Jones, R., Swann, C., Christian, H., Sherring, J., Shilton, T. \& Okely, A. (2020). Exploring \\ stakeholders' perceptions of the acceptability, usability, and dissemination of the australian 24-hour \\ movement guidelines for the early years. Journal of Physical Activity and Health, 17 (1), 120-125.

\section{Authors} \\ Rebecca M. Stanley, Rachel A. Jones, Christian F. Swann, Hayley Christian, Julie Sherring, Trevor Shilton, \\ and Anthony D. Okely
}


1 Full Title: Exploring stakeholders' perceptions of the acceptability, usability and

2 dissemination of the Australian 24-hour Movement Guidelines for the early years

3

4 Running Head: Stakeholders perceptions of Movement Guidelines

5

6 Manuscript Type: Original Research

7

8 Key Words: Physical activity, Recommendations, Young Children

9

10 Abstract Word Count: 185

11

12 Manuscript Word Count: 4934

13

14 Date of Manuscript Submission: 5/01/2019

15

16

17

18 


\section{ABSTRACT}

2 Background: Australian 24-hour Movement Guidelines for the Early Years were

3 recently developed. To maximise uptake of the guidelines, perceptions of key

4 stakeholders were sought.

5

6 Methods: 35 stakeholders (11\% Aboriginal or Torres Strait Islander descent)

7 participated in focus groups or key informant interviews. Stakeholders included

8 parents of children aged 0-5 years, early childhood educators, and health and policy

9 professionals, recruited using convenience and snowballing techniques. Focus groups

10 and interviews were audio-recorded and transcribed verbatim. Data were analysed

11 inductively using thematic analysis.

13 Results: There was general acceptance of the Movement Guidelines. The stakeholders

14 suggested that the Movement Guidelines were highly aspirational and needed to be

15 carefully messaged so parents did not feel guilty if their child was not meeting them.

16 Stakeholders identified that the messaging needed to be culturally appropriate and

17 visually appealing. Dissemination strategies differed depending on the stakeholder.

19 Conclusions: Seeking stakeholder perceptions is an important process in the

20 development of national Movement Guidelines. This study successfully examined

21 stakeholders' perceptions regarding the acceptability, usability and dissemination of

22 the Australian 24-hour Movement Guidelines. Effective and innovative strategies for

23 maximising compliance and uptake of the Movement Guidelines should be

24 prioritised. 


\section{BACKGROUND}

2 There is considerable evidence demonstrating the benefits of adequate physical

3 activity, low levels of sedentary behaviour and adequate sleep for health among

4 children, including young children (birth to 5 years) (referred to here as the Early

5 Years ${ }^{1-3}$. In recent years, there has been a shift away from exploring these

6 behaviours independently to examining the continuum of movement behaviours

7 across a 24-hour period ${ }^{2,4,5}$. This shift acknowledges the importance of movement

8 behaviours across a whole day and how these behaviours integrate with each other to

9 influence health and developmental outcomes for children ${ }^{4}$. In 2017, Canada and

10 Australia developed and co-released 24-hour Movement Guidelines for the Early

11 Years ${ }^{6}$. New Zealand, South Africa, and the WHO used a 24-hour approach in the

12 development of their guidelines which were released 2017, 2018, and 2019,

13 respectively ${ }^{7-9}$. Other countries, such as USA and UK have recently released similar

14 guidelines for the early years, however these only provide guidance on physical

15 activity.

16

17 Despite the known importance of public health related guidelines, such guidelines are

18 only effective if key stakeholders are aware of and use them ${ }^{10}$. Therefore, one of the

19 key steps in the development of the Australian 24-hour Movement Guidelines was to

20 engage key stakeholders in the evaluation of the appropriateness of the Movement

21 Guidelines and identification of knowledge translation strategies ${ }^{10-12}$. Understanding

22 the needs of key stakeholders can help with maximising compliance and uptake of the

23 Movement Guidelines ${ }^{13,14}$ and ultimately the health of the target population ${ }^{10,15}$. 
1 Only a few studies have reported consultations with stakeholders to explore needs,

2 values or recommended dissemination strategies for public health related guidelines,

$3{ }^{15,16,17}$, however only one study has focused on the early years ${ }^{17}$. Therefore, the aim of

4 this study was to examine stakeholders' perceptions of the Australian 24-hour

5 Movement Guidelines for the Early Years, specifically their acceptability, usability

6 and dissemination strategies during the development stage and prior to release of the

7 Guidelines.

9 METHODS

$10 \quad$ Stakeholders

11 Thirty-five individuals participated in five focus groups (3-9 stakeholders per focus 12 group) and five key informant interviews (1 participant per interview). The average 13 age of stakeholders was 35.1 years (range: $17-56$ years; $\mathrm{SD}=9.1$ ) and 33 of the 35

14 stakeholders identified as female. The majority of stakeholders (97\%) reported

15 English as their predominant language spoken at home, $89 \%$ had completed a post-

16 secondary education and $11 \%$ self-identified as being of Aboriginal or Torres Strait

17 Islander descent.

19 Stakeholders in the focus groups consisted of parents/caregivers from diverse

20 socioeconomic and cultural backgrounds (e.g. high and low socio economic status

21 (SES), Indigenous populations, culturally and linguistic diverse populations), and

22 early childhood educators and health workers (including those who work with

23 children with additional needs). Each focus group comprised stakeholders of similar

24 backgrounds (e.g. parents and educators who identified as Indigenous $n=5$, health

25 professionals who worked with families and children with additional needs $n=3$, 
1 parents from low SES communities, $n=3$, parents from mid to high SES communities

$2 \mathrm{n}=9$, parents from culturally and linguistically divers communities $\mathrm{n}=10$ ). Key

3 informant interviews were held with a paediatrician, a general practitioner with a

4 diploma in Child Health, a paediatric physiotherapist, a representative of the

5 organisation leading the implementation of the National Quality Framework for early

6 childhood education and care services in Australia, and a representative of a not-for-

7 profit organisation representing long-day care owners and operators.

9 Key stakeholders were recruited through existing networks of the Guideline

10 Development Group for the Australian 24-hour Movement Guidelines for the Early

11 Years (referred from here as Movement Guidelines), as well as through snowballing

12 techniques ${ }^{18}$. Informed consent was obtained at the beginning of the interviews and

13 focus groups, followed by a short demographic questionnaire. This study received

14 ethical approval from the University of Wollongong Human Research Ethics

15 Committee (HE 2017/164).

Data collection

18 Data were collected through semi-structured one-on-one telephone interviews and

19 face-to-face focus groups. Each stakeholder was given a plain-text printed copy of the

20 draft Movement Guidelines, which included the preamble and the guideline document

$21{ }^{19}$, and were asked to read and familiarising themselves with the documents prior to

22 the discussion. The open-ended discussions focused on: (i) acceptability of the

23 Movement Guidelines; (ii) perceived importance and clarity of the Movement

24 Guidelines and preamble; (iii) facilitators and barriers to implementation and

25 dissemination; and (iv) dissemination and implementation strategies ${ }^{16,17}$. Focus 
1 groups and key informant interviews were conducted from late-May to mid-

2 September 2017 across three States in Australia: New South Wales (NSW), Victoria

3 (Vic) and Western Australia (WA). Focus groups and interviews lasted on average 40

4 minutes and were conducted by four researchers (RMS, RAJ, HC and JS). Data were

5 collected until saturation was perceived by the research team, that is, no new

6 information emerged from the final two interviews ${ }^{20}$.

7

8 Analysis

9 The audio-recordings from the interviews and focus groups were transcribed

10 verbatim. To maintain confidentiality, stakeholder names were replaced using the

11 terms Parent, Educator, Health Professional, Policy Professional and Researcher,

12 along with the key informant interview (Int) or focus group (FG) number and

13 corresponding Australian State (NSW, WA, Vic) in which the discussion group was

14 held (e.g. Parent, FG1, NSW). Stakeholders are referred-to by these codes below. In

15 line with the aims of this study, acceptability, usability, and dissemination were

16 identified a priori as the key areas of focus. Then, for each of these aspects, the

17 transcripts were analysed inductively using thematic analysis ${ }^{21}$. This process

18 followed the six phases recommended by Braun et al. ${ }^{21}$ : (i) familiarisation with the

19 data; (ii) deriving codes from the data (i.e., succinct labels that captured key analytical

20 ideas); (iii) searching for themes (i.e., clusters of similar codes which make up a

21 broader level of meaning); (iv) reviewing themes; and (v) defining and naming

22 themes, before (vi) producing the report (see below).

23

24 Strategies were implemented to ensure trustworthiness (e.g., quality and rigour) of the 25 analysis. Peer debrief ${ }^{22}$ was conducted throughout the study via regular formal and 
1 informal meetings between the lead researcher and each member of the research team

2 (collectively and individually). Furthermore, a team approach to analysis was

3 adopted, which enabled two co-authors to act as 'critical friends' ${ }^{23}$. That is, the lead

4 researcher (RMS) and two co-authors (RAJ and CS) read each transcript

5 independently. Then, all three authors shared their interpretations, and offered critical

6 feedback to the lead researcher (RMS), in order to encourage reflexivity, explore

7 multiple/alternative interpretations, and ultimately enhance plausibility of the

8 resulting analysis ${ }^{23}$.

10 Results

11 Three broad themes were identified in the analysis of the focus group and key

12 informant interview transcripts: (1) acceptability, (2) usability and (3) dissemination.

\section{Acceptability}

15 There was a general acceptance among all stakeholders that the Movement Guidelines

16 were easy to understand and follow. All stakeholders favourably supported the

17 integration of behaviours (i.e. physical activity, sedentary behaviour and sleep) and

18 the division of the behaviours based on ages (i.e., infants, toddlers and pre-schoolers).

19 Educators and other professionals suggested that allocating a 'time' length to each

20 behaviour (e.g., three hours of physical activity and less than one hour of sedentary

21 behaviour) was also beneficial as "this would provide a clear guide for parents"

22 (Policy Professional, Int1, NSW). Stakeholders who were professionals suggested that

23 the Movement Guidelines filled a gap in the information currently available and

24 provided an opportunity to guide practice where guidelines and policy on early

25 movement behaviour was currently limited. As one professional suggested: 
23 The second area of refinement identified by the stakeholders was the visual appeal of “...They [early childhood (ECEC) educators] need something like this because they're not thinking about physical activity. They're thinking about sleep certainly, and they're thinking about the types of food children are eating and how much time they're spending outside, but they're not thinking about that holistically with regards to physical activity." (Policy Professional, Int2, WA)

Despite an overall acceptance of the Movement Guidelines, there were a few key areas that stakeholders suggested needed to be refined to ensure optimal uptake. The first area was the removal of movement behaviour-related jargon (e.g., 'energetic play', 'cognitive development', 'cardiorespiratory', 'sleep hygiene' etc). All stakeholders (including the health and policy professionals) suggested that such words may be difficult to understand for the general population. Stakeholders were particularly concerned about the understanding of such words for those from lower socio-economic backgrounds or those whose first language was not English: ...So I just think the language is really [hard], [it] runs the risk of people switching off and not even paying [attention], like not being able to understand it, avoiding going on with it...they'll read down that preamble and get to some of that stuff, some of the language and just go 'oh, I don't even understand this' and just put it away.... or not read on anymore... (Policy Professional, Int1, NSW)

the Movement Guidelines. For the purposes of the consultations, stakeholders were provided with a plain-text draft of the Movement Guidelines as a black and white 
1 Word document. Stakeholders understood that this was a draft document, however

2 they were eager to stress that the layout of the final document was critically important

3 for uptake and application in practice. Stakeholders suggested that the presentation of

4 the Movement Guidelines needed to be appealing, inviting and culturally appropriate

5 and have an emphasis on artwork and visuals rather than the text. Those from the

6 lower socio-economic or Indigenous groups specifically emphasised this concern and

7 suggested that parents would not be interested in such a document unless the

8 presentation was prioritised. One parent clearly summarised this sentiment: “...but to

9 give them a pamphlet like that, honestly, I don't even think it would get read; they'd

10 just look at it and go, "Hm, whatever..." (Parent, FG1, NSW)

12 Although all stakeholders suggested that the times allocated to the different

13 behaviours were helpful as a guide, parents and educators were concerned that the

14 prescription of such behaviours may result in parents feeling guilty if they could not

15 meet the recommended times; and not meeting the guidelines could reflect poorly on

16 their parenting style. Although all parent and educator stakeholders expressed these

17 concerns, they were predominantly expressed by Indigenous parents or parents from

18 lower socio-economic demographics:

19 "Oh my God, get to sleep, what am I doing wrong?", and they'll take it 20 personally, and go to all these different places and be like "Oh my God, what 21 am I doing wrong, they're not sleeping for the minimum of 10 hours" (Parent, $F G 2, W A)$ 


\section{7}

need time", you know, they might feel a bit helpless as to how they're going to achieve that (Parent, FG2, WA)

\section{Usability}

The Movement Guidelines were developed for Early Years stakeholders, with the primary end users being parents and early childhood educators. Parents accepted the content of the Movement Guidelines, however they suggested a number of barriers that were likely to impede their usability. Time and poor weather were common barriers mentioned. Competing interests of older siblings was also mentioned as a potential barrier. For example, parents suggested that it would be more difficult for children from larger families to participate in the prescribed movement behaviours as younger children in such families are often driven around to other extra curricula activities and often provided with a piece of technology to occupy themselves while the activity was taking place: "They [the older children] go to gymnastics and you can't let them run around, you've got to sit. So we have to do iPad, which is fine, he [younger child not involved in gymnastics] loves it." (Parent, FG3, NSW)

The availability of technology-based devices within the home environment was also

suggested to be a significant confounder in terms of usability of the Movement

Guidelines. Parents suggested that technology is often the first point of entertainment

1 for young children and is regularly used as an 'electronic babysitter', rather than

2 involving children in other activities such as energetic play. A parent from the high

socio-economic focus group succinctly summarised these barriers:

Enough time in the day. I think the biggest challenge now is time, well a lot of 5 families are both working parents. Times are changing and everyone's 
running in the door and in and out. There's so much sport and outside stuff of school...I think for our family it's the competing needs of various members of the family. That is an issue (Parent, FG3, VIC)

To aid the usability of the Movement Guidelines, parents suggested providing detailed examples of what these behaviours looked like in practice, for example, "what energetic play looked like" and "examples of how to reduce screen time" (Parent, FG1, NSW). Many parents had incorrect misconceptions of the movement behaviours. For example, a number of parents suggested that physical activity should involve children participating in costly structured physical activity, organised games or "making children running laps around an oval" (Parent, FG1, NSW). The provision of specific and detailed examples could potentially alleviate such misconceptions related to the movement behaviours.

In alignment with the suggestions from parents, early childhood educators also suggested that the usability of the Movement Guidelines would be influenced by the weather and time. Educators suggested that their time was limited due to the high demands of administration within ECEC settings, which often resulted in other activities being compensated. In addition, educators suggested that the usability of the Movement Guidelines would be significantly influenced by the underpinning philosophy of the ECEC setting. In Australia, ECEC services are guided by a number of different philosophies and curriculums, many of which prioritise the cognitive and social/emotional key learning domains rather than the physical domain: “...And for the childcare centres, I know they have certain parts of the curriculum they need to 
1 adhere to, and if the curriculum's demanding, then they put more focus on academic

2 rather than physical activity" (Educator, Int, NSW)

$4 \quad$ Similar to parents, early childhood educators suggested that the addition of several

5 broad reaching examples would be helpful in optimising usability, for example, use of

6 music and flashcards. Specific examples of how the movement behaviours could

7 realistically be incorporated in their day to day planning as well as integrated into

8 other curriculum areas was also thought to be critical for maximising the usability.

9 Some educators suggested that being accountable for implementation of the

10 movement behaviours would be helpful, and thus practical ideas of how to include

11 these behaviours in their planning was suggested to be important. As one educator 12 suggested: "My way of thinking with this is that if we were to put this in our QIP 13 [Quality Improvement Plan], and they come through and they say "Well, how are you 14 doing that?... we've got no documentation." (Educator, FG4, WA)

16 Meaningful collaborations between parents and their children, parents/families and 17 educators and between educators and the children in their care were identified as 18 being critical for high usability. Professionals and parents involved in the stakeholder 19 consultations suggested that the usability of the Movement Guidelines would be 20 enhanced if parents were intentional in 'role modelling' the correct behaviours and 21 being 'play partners' (i.e., joining in). One parent explained the impact of this on her 22 family:

23 Michael* and I joined in the other night. During family time, for 20 minutes, 24 Simon's* rule was that we had to run, George's* rule was that we had to run laps around the house, holding our card and see who the winner was. And we 
had a ball, Michael* and I, running. And I got breathless. It was vigorous. (Parent, FG3, VIC)* Names are pseudonyms

4 Educators emphasised the importance of consistent messaging between the ECEC

5 environment and the family environment. Ongoing education and awareness from

6 both early childhood educators and parents of the importance and application of the

7 movement behaviours was highlighted as necessary. There was concern from the

8 stakeholders that it may be easier to educate educators in this regard but harder to

9 educate families, for example:

But there needs to be more education around it. It's easy to educate professionals who work with young children, but it's harder to educate families obviously. (Policy professional, Int, WA) “...it would be harder to try and get the parents on board with it..." (Educator, FG4, WA)

\section{Dissemination}

Stakeholders identified a range of dissemination options. These were largely groupspecific (i.e., dissemination options for parents were different from those for

22 avenues across all groups. Parents suggested it would be most appropriate to receive

23 information about the Movement Guidelines from health professionals and health

24 services (such as child health nurses, support services). They suggested that they

25 regularly consult with such professionals and would be willing to receive information 
1 from such people. They also suggested that social media, apps/websites (e.g. Raising

2 Children's Network) and community groups (e.g., mother's groups and play-based

3 groups) would be key places for dissemination. Parents from the Indigenous parent

4 group suggested face-to-face communication and discussions (e.g., yarn groups: small

5 groups of people who gather to talk (or yarn) about a certain issue) would be helpful

6 as these are culturally appropriate strategies for communicating information. As one

7 parent suggested: “yes...have a yarn-up and communicate."

9 Educators and professionals suggested that dissemination through evidenced-based

10 websites and social media would be appropriate. Professionals suggested that they

11 often referred to online resources for additional information and suggested that the 12 information pertaining to the Movement Guidelines would be helpful to have online.

13 Educators suggested that further education pertaining to the Movement Guidelines

14 could be best disseminated through staff meetings whilst professionals suggested that

15 receiving information about the Movement Guidelines through their specific

16 professional societies, in-service courses and conferences would be most helpful.

\section{DISCUSSION}

19 This study explored the perceptions of key stakeholders and end users (parents, early

20 childhood educators, policy and health professionals) regarding the acceptability,

21 usability and dissemination of the Australian 24-hour Movement Guidelines for the

22 Early Years (birth to 5 years). In summary, there was general acceptance of the

23 Movement Guidelines among all stakeholders, however views relating to usability

24 and dissemination varied between stakeholder groups. The general acceptance of the

25 integrated behaviour model from all stakeholders was similar to that reported by the 
1 stakeholder consultations for the Canadian 24-hour Movement Guidelines for the

2 Early Years ${ }^{17}$. This finding is significant since high acceptability underpins the

3 quality of the content and in turn the potential impact of guidelines ${ }^{10,24}$.

5 In this study there was a discourse between professionals, educators and parents in

6 relation to the usability of the Movement Guidelines. This was particularly noticeable

7 in relation to the prescribed durations (e.g., 60 minutes of energetic play for preschool

8 aged children etc.) for the behaviours. The health professionals, on the whole,

9 suggested that the times were appropriate and recognised that such amounts would be

10 needed for positive child health-related outcomes. In contrast, parents and educators,

11 especially parents from lower socio-economic and Indigenous groups, suggested that

12 the messaging around the recommended times children should spend in each

13 behaviour could stimulate "feelings of guilt". The reasons provided (e.g., time, work/

14 family commitments) for not being able to achieve the Movement Guidelines are

15 similar to those previously identified as key barriers to health behaviour change

16 among Indigenous and low socio-economic populations ${ }^{17,25}$. These findings are of

17 concern as it was suggested that the parent guilt could potentially result in families not

18 trying to adhere to the Movement Guidelines. To alleviate such potential feelings of

19 guilt it is important that the preceding messaging and advertising associated with the

20 Movement Guidelines is appropriate and clearly highlights that the recommendations

21 in the Movement Guidelines are a guide, with the main message being that for greater

22 health, 24 hours should include some screen time and lots of physical activity and

23 even more sleep ${ }^{19}$. 
1 The discourse between parents, early childhood educators and professionals did not

2 seem to be as definitive in the 24-hour Movement Guideline Canadian stakeholder

3 consultations. The Canadian stakeholder consultations similarly involved a diverse

4 group of stakeholders of whom the majority had completed post-secondary education

$5 \quad{ }^{17}$. However, the current study included more Indigenous perspectives compared to

6 the Canadian sample ${ }^{17}$. The higher ratio of Indigenous parents and those from lower

7 socio-economic backgrounds in the current study may have resulted in the more

8 obvious discourse. With a growing demographic of families and early childhood

9 educators from culturally and linguistically diverse backgrounds in Australia ${ }^{26}$,

10 language, wording and design considerations should be a priority.

12 Stakeholders in the current study identified a comprehensive list of potential

13 dissemination resources, avenues and key messengers. However, these suggestions

14 may only be effective if they are acted upon as part of a comprehensive knowledge

15 translation and dissemination strategy. There may be little point in investing in the

16 development of new guidelines or even having public health related guidelines if they

17 are not done in conjunction with a well-resourced and longer term dissemination and

18 implementation plan. Therefore resources should be made available for involving key

19 stakeholders in the design and implementation of the Movement Guidelines

20 dissemination strategies, otherwise efforts to maximise guideline reach and uptake

21 may be limited ${ }^{17}$. The development and dissemination of simple infographics may

22 provide an appropriate and effective initial first step.

24 Irrespective of dissemination method/s appropriate dissemination should be

25 underpinned by consistent messaging and include a collaborative approach. That is, 
1 parents/caregivers should be exposed to the same consistent message from different

2 sources, multiple times throughout their child's Early Years. Effective dissemination

3 needs to involve parents/caregivers hearing, seeing and discussing the Movement

4 Guidelines regularly, for example, in the day-to-day life of the family, in social/peer

5 networks, at ECEC settings, when interacting with health and other professionals

6 face-to-face and online. Given that parents of young children are one of the largest

7 users of social media ${ }^{27}$, innovative social media messages maybe an appropriate and

8 effective dissemination avenue. Population level effective social marketing can be

9 expensive; however, given the breadth of social marketing options available to date,

10 some innovative social marketing strategies may be effective and not require

11 extensive resources.

13 It is critical that parents, early childhood educators and health professionals, advocate

14 for the dissemination of the Movement Guidelines. Collaborations between

15 stakeholders are needed for effective dissemination and in turn effective uptake of the

16 Movement Guidelines. It was beyond the scope of this study to further investigate

17 how such collaborations could be established and developed to ensure effective

18 dissemination and implementation, however this is an important area to consider in

19 future research on implementation of the Movement Guidelines among the public. If a

20 coordinated approach is applied ${ }^{17}$, then it is more likely to result in positive

21 modification of young children's movement behaviours ${ }^{28,29}$.

\section{Strengths and limitations}

24 This study successfully captured the perceptions of a diverse range of stakeholders

25 including Indigenous groups and low socio economic groups. Stakeholders were 
1 recruited from three states across Australia. While attempts were made to include

2 stakeholders from other areas in Australia (e.g., Queensland and Northern Territory)

3 this was not possible in the given timeframe. Additionally, the sample size for this

4 study was lower than Canadian Stakeholder consultations yet included a greater

5 number of Indigenous stakeholders. Despite not having all States and Territories

6 represented and the sample size being smaller, saturation of themes was reached. The

7 number of participants recruited to some of the focus groups was low. This may have

8 impacted the diversity in group ideas and themes, More resources and a longer period

9 for data collection would have been beneficial for maximising optimal participant

10 numbers in each focus group.

\section{CONCLUSION}

13 This was the first study to engage stakeholders in the development of the Australian

14 24-hour Movement Guidelines for the Early Years. The study successfully examined

15 stakeholders' perceptions regarding the acceptability, usability and dissemination of

16 these 24-hour Movement Guidelines. A number of key elements were identified,

17 which, if considered would significantly impact the usability of the Movement

18 Guidelines. If the 24-hour Movement Guidelines for the Early Years are appropriately

19 integrated and uptake is maximised, such Movement Guidelines have significant

20 potential to change the physical activity, health and development trajectory of young

21 children. 


\section{REFERENCES}

1. Carson V, Lee EY, Hewitt L, et al. Systematic review of the relationships between physical activity and health indicators in the early years ( $0-4$ years). BMC public health. 2017;17(Suppl 5):854.

2. Chaput JP, Gray CE, Poitras VJ, et al. Systematic review of the relationships between sleep duration and health indicators in the early years (0-4 years). BMC public health. 2017;17(Suppl 5):855.

3. Poitras VJ, Gray CE, Janssen X, et al. Systematic review of the relationships between sedentary behaviour and health indicators in the early years (0-4 years). BMC public health. 2017;17(Suppl 5):868.

4. Chaput JP, Carson V, Gray CE, Tremblay MS. Importance of all movement behaviors in a 24 hour period for overall health. International journal of environmental research and public health. 2014;11(12):12575-12581.

5. Kuzik N, Poitras VJ, Tremblay MS, Lee EY, Hunter S, Carson V. Systematic review of the relationships between combinations of movement behaviours and health indicators in the early years (0-4 years). BMC public health. 2017;17(Suppl 5):849.

6. Tremblay MS, Chaput JP, Adamo KB, et al. Canadian 24-Hour Movement Guidelines for the Early Years (0-4 years): An Integration of Physical Activity, Sedentary Behaviour, and Sleep. BMC public health. 2017;17(Suppl 5):874.

7. $\quad$ http://www.laureus.co.za. http://www.laureus.co.za/wpcontent/uploads/2019/04/EYMG-tips_ECD-practitioners-ONLINE.pdf. Published 2018. Accessed May 2019. 
1 8. $\quad$ https://www.health.govt.nz/system/files/documents/publications/active-playguidelines-for-under-fives-may17.pdf. Published 2017. Accessed May 2019.

39 9. http://www.who.int. https://www.who.int/news-room/detail/24-04-2019-togrow-up-healthy-children-need-to-sit-less-and-play-more. Published 2019. Accessed May 2019.

6 10. Brouwers MC, Kho ME, Browman GP, et al. AGREE II: advancing guideline development, reporting, and evaluation in health care. Prev Med. 2010;51(5):421-424.

9 11. Tremblay MS, Haskell W. From Science to Physical Activity Guidelines. In: Physical activity and health. 2nd ed. Champaign: Human Kinetics; 2012:359378.

12. Lavis JN, Robertson D, Woodside JM, McLeod CB, Abelson J, Knowledge Transfer Study G. How can research organizations more effectively transfer research knowledge to decision makers? Milbank Q. 2003;81(2):221-248, 171 -

14. Graham ID, Logan J, Harrison MB, et al. Lost in knowledge translation: time for a map? The Journal of continuing education in the health professions. 2006;26(1):13-24.

15. Gagliardi AR, Brouwers MC, Palda VA, Lemieux-Charles L, Grimshaw JM. How can we improve guideline use? A conceptual framework of implementability. Implementation science : IS. 2011;6:26. 
1 16. Faulkner G, White L, Riazi N, Latimer-Cheung AE, Tremblay MS. Canadian

24-Hour Movement Guidelines for Children and Youth: Exploring the perceptions of stakeholders regarding their acceptability, barriers to uptake, and dissemination. Applied physiology, nutrition, and metabolism $=$ Physiologie appliquee, nutrition et metabolisme. 2016;41(6 Suppl 3):S303310.

17. Riazi N, Ramanathan S, O'Neill M, Tremblay MS, Faulkner G. Canadian 24hour movement guidelines for the early years (0-4 years): exploring the perceptions of stakeholders and end users regarding their acceptability, barriers to uptake, and dissemination. BMC Public Health. 2017;17(Suppl 5):841.

18. King N, Horrocks C. Interviews in qualitative research. SAGE; 2010.

19. Okely $\mathrm{AD}$, Ghersi $\mathrm{D}$, Hesketh $\mathrm{KD}$, et al. A collaborative approach to adopting/adapting guidelines - The Australian 24-Hour Movement Guidelines for the early years (Birth to 5 years): an integration of physical activity, sedentary behavior, and sleep. BMC public health. 2017;17(Suppl 5):869.

20. Saunders B, Sim J, Kingstone T, et al. Saturation in qualitative research: exploring its conceptualization and operationalization. Quality \& quantity. 2018;52(4):1893-1907.

21. Braun V, Clarke V, Terry G. Thematic Analysis. In: Rohleder P, Lyons AC, eds. Qualitative research in clinical and health psychology. Macmillan International Higher Education; 2014.

22. Creswell JW, Miller DL. Determining validity in qualitative inquiry. Theor Pract. 2000;39(3):124-130. 
1 23. Smith B, McGannon K. Developing rigor in qualitative research: problems and opportunities within sport and exercise psychology. International Review of Sport and Exercise Psychology. 2018;11(1):101-121.

24. Kredo T, Bernhardsson S, Machingaidze S, et al. Guide to clinical practice guidelines: the current state of play. International journal for quality in health care : journal of the International Society for Quality in Health Care. 2016;28(1):122-128.

25. Carson V, Clark M, Berry T, Holt NL, Latimer-Cheung AE. A qualitative examination of the perceptions of parents on the Canadian Sedentary Behaviour Guidelines for the early years. Int J Behav Nutr Phys Act. 2014;11:65.

26. Ortlipp M, Nuttall J. Supervision and Assessment of the Early Childhood Practicum: Experiences of Pre-service Teachers Who Speak English as a Second Language and Their Supervising Teachers Australasian Journal of Early Childhood. 2011;36(2):87-94.

27. Duggan M, Lenhart, A., Lampe, C., \& Ellison, N. Parents and Social Media. Published 2015. Accessed.

28. Latimer-Cheung AE, Rhodes RE, Kho ME, et al. Evidence-informed recommendations for constructing and disseminating messages supplementing the new Canadian Physical Activity Guidelines. BMC public health. 2013;13:419.

29. Michie S, van Stralen MM, West R. The behaviour change wheel: a new method for characterising and designing behaviour change interventions. Implementation science : IS. 2011;6:42. 
Stakeholders perceptions of Movement Guidelines

2 FUNDING SOURCE

3 This research was funded by the Commonwealth Department of Health, Australia. 